\section{Oxygen Desaturation in Patients During Commercial Jet Aircraft Repatriation}

\author{
Mahomey JF, Pigman EC, Hunt DM \\ Department of Emergency Medicine, George Washington \\ University Medical Center, \\ Washington, District of Columbia, USA
}

Purpose: Individuals becoming ill or injured at locations remote from their homes frequently require urgent air repatriation for definitive and convalescent care. This study was initiated anticipating that patients with cardiopulmonary or other severe disease processes would be subject to clinically significant oxygen desaturation at standard commercial cruising altitudes.

Method: This was a prospective, case-controlled, observational study. Fifty consecutive patients, for whom pulse oximetry was available, required medical accompaniment during repatriation on commercial air carriers. Repatriation occurred an average of 14 days after onset of the index symptom. Specific diagnoses included recent myocardial infarction and congestive heart failure $(n=31)$, hip fractures $(n=9)$, and other diagnoses including recent stroke and/or pneumonia $(n=10)$. The patient's medical attendant, traveling companion, and flight attendant served as controls. All $p$-values are 2-tailed and reported after Bonferroni's correction.

Results: No symptoms occurred during the 50 repatriations. The mean change from pre-flight to in-flight oxygen saturation as measured by pulse oximetry was $3.4 \%$ in Cardiacs, $5.7 \%$ in Fractures, $2.3 \%$ in Others, and $1.9 \%$ in Controls (ANOVA, $p=$ .0056). Older ages correlated with greater change in oxygen saturation $(p=.0035)$. Additional variables not significantly related to changes in oxygen saturation were gender, pre-flight pulse oximetry, tobaccoism, prior pulmonary disease, and the effective cabin cruising altitude.

Conclusions: Older travelers and patients with acute cardiac disease or recent hip fractures show the greatest oxygen desaturation during commercial jet aircraft repatriation. Pre-flight pulse oximetry does not predict the amount of decrease in inflight oxygen saturation as measured by pulse oximetry.

\section{Cardiac Arrest Epidemiology: Low Income Increases Cardiac Arrest Rate}

\author{
Feero SM, ${ }^{*+}$ Stevens PJ, ** Hedges $\mathrm{JR}^{+}$ \\ * St. Peter Hospital, Olympia, Washington, USA \\ ** Oregon Health Sciences University, Emergency Department, \\ Portland, Oregon, USA \\ + Oregon Health Sciences University, \\ Department of Emergency Medicine, \\ Portland, Oregon, USA
}

Hypothesis: Median income is associated inversely with metropolitan cardiac arrest rate.

Methods: Retrospective analysis of 363 victims of primary cardiac arrest using census tract data for a metropolitan area (516,601 population; 200 square miles). The city census tracts were separated into seven geographic regions based upon natural boundaries. Regional characteristics were determined from 1990 census-tract data and evaluated for relative risk (RR) of arrest as a function of arrest site and victim residence site. Associations with other demographic parameters were sought using the regional Z-score for arrest rate after adjustment for victim age and gender.

Results: Patient age and gender were independent determinants of RR for cardiac arrest. After adjustment for age and gender, one region had a larger RR for primary cardiac arrest $(\mathrm{RR}=1.9$ and 1.7 ; for arrest site and victim residence site, respectively). Among the regions, there was no association of cardiac arrest rate with percentage of the population that was Caucasian or with the population density. However, median income was associated inversely with the adjusted cardiac arrest rate $(p=.012)$. When the census tracts with the 20 lowest median incomes were compared with the census tracts with the 20 highest median incomes, the RR for cardiac arrest with low income was $2.7(95 \% \mathrm{CI}=1.7-4.2$ for arrest site and $1.8(95 \%$ $\mathrm{CI}=1.1-2.9)$ for victim residence.

Conclusions: Population age and gender data generally predicted cardiac arrest rates in our urban setting although one outlier region was found. A strong inverse relationship was found between median income and cardiac arrest rate, suggesting cardiac arrest prevention efforts should be targeted for low-income neighborhoods. 